Gut and Liver, Vol. 11, No. 5, September 2017, pp. 648-654

\title{
In Vitro Activity of Diphenyleneiodonium toward Multidrug-Resistant Helicobacter pylori Strains
}

\author{
Jun-Won Chung ${ }^{1}$, Su Young Kim ${ }^{1}$, Hee Jung Park ${ }^{2}$, Chang Su Chung ${ }^{1}$, Hee Woo Lee ${ }^{1}$, Sun Mi Lee ${ }^{3}$, Inki Kim ${ }^{3}$, Jhang Ho Pak ${ }^{3}$,
} Gin Hyug Lee ${ }^{2}$, and Jin-Yong Jeong ${ }^{3}$

${ }^{\prime}$ Division of Gastroenterology, Department of Internal Medicine, Gachon University Gil Medical Center, Incheon, Departments of ${ }^{2}$ Gastroenterology and ${ }^{3}$ Convergence Medicine and Asan Institute for Life Sciences, Asan Medical Center, University of Ulsan College of Medicine, Seoul, Korea

Background/Aims: The increased resistance of Helicobacter pylori to antibiotics has increased the need to develop new treatments for this bacterium. The aim of our study was to identify new drugs with anti-H. pylori activity. Methods: We screened a small molecule library-the library of pharmacologically active compounds (LOPAC), which includes 1,280 pharmacologically active compounds-to identify inhibitors of $H$. pylori growth. The minimal inhibitory concentrations (MICs) of antibiotics against multidrug-resistant $H$. pylori strains were determined using the agar dilution method. Results: We identified diphenyleneiodonium (DPI) as a novel anti$H$. pylori agent. The MIC values for DPI were $<0.03 \mu \mathrm{g} / \mathrm{mL}$ against all tested $H$. pylori strains. DPI also exhibited strong antibacterial activity against common gram-negative and gram-positive pathogenic bacteria. Conclusions: DPI may be a candidate anti-H. pylori drug for future development. (Gut Liver 2017;11:648-654)

Key Words: Helicobacter pylori; Diphenyleneiodonium; Drug resistance, multiple; Anti-bacterial agents; Minimal inhibitory concentration

\section{INTRODUCTION}

Helicobacter pylori infection is the main cause of peptic ulcer disease, mucosa-associated lymphoid tissue lymphoma, and gastric cancer. The eradication of $H$. pylori has been shown to dramatically decrease the recurrence of peptic ulcer disease, including gastric and duodenal ulcers. ${ }^{1}$ Currently, it is estimated that around 50\% of the world's population has this bacterium. ${ }^{2}$ Hence, eradicating this organism is of significant clinical importance. According to various guidelines published since 1993, the first-line choice of treatment for $H$. pylori eradication consists of conventional triple therapy, which includes a proton pump inhibitor (PPI), clarithromycin, and amoxicillin for 7 to 14 days. Over the past few years, however, the efficacy of conventional triple therapy has decreased and now demonstrates eradication rates of less than $80 \%{ }^{3,4}$ This decrease is mainly due to the emergence of clarithromycin-resistant $H$. pylori strains.

To improve first-line treatments for $H$. pylori, a four-drug treatment (including metronidazole), with sequential and concomitant bismuth-based quadruple therapy for 7 to 14 days, was introduced. In many previous studies, this regimen has demonstrated a better eradication rate than that of conventional triple treatment as the first-line treatment. ${ }^{5-7}$ However, all of these treatments include metronidazole, which has been conventionally used as a second-line treatment. Hence, there is concern that these four-drug regimens could worsen antibiotic resistance and decrease the second-line eradication rate. Another drawback to these complex regimens is that they increase both the cost of therapy and patient noncompliance. In addition, quinolone is popularly used as a second-line treatment after a four-drug treatment regimen. ${ }^{8}$ The quinolone resistance rate also has dramatically increased in recent years. ${ }^{9}$

Various methods have recently been introduced to overcome the drawbacks of four-drug treatment and fluoroquinolonecontaining therapy. Rifabutin-containing therapy, probiotics, and tailored therapy are alternative methods. ${ }^{10,11}$ However, these

Correspondence to: Jin-Yong Jeong ${ }^{\mathrm{a}}$ and Gin Hyug Lee ${ }^{\mathrm{b}}$

${ }^{a}$ Department of Convergence Medicine and Asan Institute for Life Sciences, Asan Medical Center, University of Ulsan College of Medicine, 88 Olympic-ro 43-gil, Songpa-gu, Seoul 05505, Korea

Tel: +82-2-3010-4105, Fax: +82-2-3010-4182, E-mail: jyjeong@amc.seoul.kr

bepartment of Gastroenterology, Asan Medical Center, University of Ulsan College of Medicine, 88 Olympic-ro 43-gil, Songpa-gu, Seoul 05505, Korea

Tel: +82-2-3010-3197, Fax: +82-2-485-5782, E-mail: jhlee409@chollian.net

Received on October 13, 2016. Revised on January 6, 2017. Accepted on February 13, 2017. Published online July 28, 2017

pISSN 1976-2283 eISSN 2005-1212 https://doi.org/10.5009/gnl16503

Jun-Won Chung and Su Young Kim contributed equally to this work as first authors.

@) This is an Open Access article distributed under the terms of the Creative Commons Attribution Non-Commercial License (http://creativecommons.org/licenses/by-nc/4.0) which permits unrestricted non-commercial use, distribution, and reproduction in any medium, provided the original work is properly cited. 
methods involve some challenges. Rifabutin causes serious complications, such as myelosuppression, and is difficult to use in countries with a high incidence of Mycobacterium tuberculosis. ${ }^{12,13}$ Probiotics are relatively invulnerable agents, but the cost of eradication has increased and they have not been clinically effective in some randomized control trials. ${ }^{14,15}$ Most importantly, the ultimate disadvantage of the various methods presented so far is that they could increase antibiotic resistance. Antibiotic resistance has been increasing gradually since $H$. pylori eradication began, and $H$. pylori will eventually become resistant to several antibiotics. ${ }^{10,16}$ This will increase the incidence of multidrug-resistant $H$. pylori, and effective antibiotic regimens will gradually disappear. Therefore, resistance to diverse antibiotics indicates the need to develop new drugs that are effective against resistant strains.

The library of pharmacologically active compounds (LOPAC) is a collection of high-quality innovative molecules, such as antibiotics, enzyme inhibitors, cell-cycle regulators, and various other substances. It is designed for the identification of novel drug discovery assay and commonly used for screening of novel agents in drug discovery fields. LOPAC includes a large number of small molecules, and small molecules have certain benefits, such as high chemical stability and simple synthesis. Additionally, small molecules registered with LOPAC are commercially available and their effects on human cells are well known. Therefore, it is easier to apply these substances in clinical practice. Many studies have demonstrated that LOPAC is useful and effective for detecting new drugs against various pathogens, such as fungi, tuberculosis, malaria, and viruses. ${ }^{17-20}$ Therefore, we utilized LOPAC as a way to identify anti-H. pylori drugs. The primary purposes of our present study were to (1) identify new chemical agents with potential anti-H. pylori activity among the 1,200 compounds included in the LOPAC Chemical Library; and (2) measure the minimal inhibitory concentrations (MICs) of these candidates against reference and resistant strains of $H$. pylori.

\section{MATERIALS AND METHODS}

\section{Bacterial strains and culture conditions}

The well-characterized ATCC 43504 H. pylori strain (ATCC, Manassas, VA, USA) was used as the reference strain for the chemical library screening assay and the initial antimicrobial susceptibility test. Twenty clinical isolates (known to be resistant to antibiotics currently used to treat $H$. pylori) and three susceptible strains were used to test the anti- $H$. pylori activity of diphenyleneiodonium (DPI; Sigma D2926; Sigma-Aldrich Co., St Louis, MO, USA). Resistant strains included single drug- and multidrug-resistant strains. Single drug-resistant strains were resistant to clarithromycin (Sigma C9742), metronidazole (Sigma M1547), levofloxacin (Sigma 28266), or amoxicillin (Sigma A8523), all of which are frequently used to eradicate $H$. pylori.
Multidrug-resistant strains were resistant to at least two of these four drugs. Antibiotic resistance was assessed according to the Clinical and Laboratory Standards Institute (CLSI) guidelines. ${ }^{21}$ $H$. pylori strains were cultured using a selective medium that contained Brucella agar (BD 211088) and 7\% defibrinated sheep blood. Incubation of the cultured isolates was performed at $37^{\circ} \mathrm{C}$ under microaerobic conditions $\left(10 \% \mathrm{CO}_{2}\right)$ for 72 hours.

\section{Screening for inhibitors of $\boldsymbol{H}$. pylori growth}

The LOPAC Chemical Library was purchased from SigmaAldrich Co. and is composed of 1,280 small pharmacologically active molecules. These chemical compounds ( $2 \mathrm{mM}$ ) were serially diluted in 96-well source plates to select the growth inhibition concentration in a $50-\mu \mathrm{L}$ volume. Each well of the 96-well microplate contained $180 \mu \mathrm{L}$ of culture medium to which $10 \mu \mathrm{L}$ of the chemical compound and $10 \mu \mathrm{L}$ of a stock solution of $10^{6}$ H. pylori ATCC 43504 bacteria/mL was added. The plates were then incubated under microaerobic conditions at $37^{\circ} \mathrm{C}$ for 72 hours to allow for bacterial growth. After 3 days, the chemical compounds that prevented the growth of $H$. pylori were regarded as candidates for anti- $H$. pylori activity and further analyzed in antimicrobial susceptibility testing.

\section{Determination of MIC}

The susceptibilities of the $H$. pylori isolates to antibiotics were examined using the serial 2-fold agar dilution method, as previously described..$^{21}$ Briefly, the bacteria were subcultured on Mueller-Hinton agar supplemented with 5\% defibrinated sheep blood for 48 hours. The bacterial suspension was adjusted to $10^{7}$ colony-forming units and directly inoculated onto each antibiotic-containing agar dilution plate. After incubation for 72 hours, the MIC of each antibiotic was determined. The MIC range for amoxicillin, clarithromycin, and DPI is 0.03125 to 32 $\mu \mathrm{g} / \mathrm{mL}$. The MIC range for metronidazole is 0.5 to $128 \mu \mathrm{g} / \mathrm{mL}$. The standard H. pylori ATCC 43504 strain was included in these susceptibility tests as a control. The resistance breakpoints for amoxicillin, clarithromycin, metronidazole, and levofloxacin were defined as $\geq 1.0,>1.0, \geq 8$ and $>1.0 \mu \mathrm{g} / \mathrm{mL}$, respectively. The MIC results were obtained from two experiments.

\section{RESULTS}

\section{Anti-H. pylori activities of DPI}

More than 50 chemical compounds from the small-molecule LOPAC library prevented any visible $H$. pylori growth, and were regarded as having an inhibitory effect on $H$. pylori. Based on currently known pharmacological applications, we excluded antibacterial and antifungal agents that are currently used in clinical practice. Anticancer drugs acting on the cell cycle, apoptosis, DNA metabolism, and phosphorylation were also excluded. We also excluded antipsychotics and antidepressants that could affect the central nervous system through unexpected 
mechanisms of action. Calcium channel blockers and calcium channel activators were also excluded due to the possibility of cardiac toxicity. Finally, we selected a promising candidate, DPI, for further analysis (Fig. 1). DPI is a nicotinamide adenine dinucleotide phosphate (NADPH) oxidase inhibitor that reduces the production of reactive oxygen species (ROS) and may reverse atherosclerosis. DPI is presently under investigation for its possible neuroprotective effects against focal cerebral ischemia, but there are no reports on its antimicrobial activity. The MIC value of DPI against the $H$. pylori ATCC 43504 strain was $<0.03$ $\mu \mathrm{g} / \mathrm{mL}$.

We further evaluated the possible anti- $H$. pylori activities of DPI using multidrug-resistant $H$. pylori strains. The MIC values of DPI for the ATCC 43504 strain and 23 clinical isolates are listed in Table 1 . The antibiotic resistance of each strain is also stated in Table 1 . The MIC value of DPI was $<0.03 \mu \mathrm{g} / \mathrm{mL}$ against all of the tested $H$. pylori strains, indicating strong antiH. pylori activity.

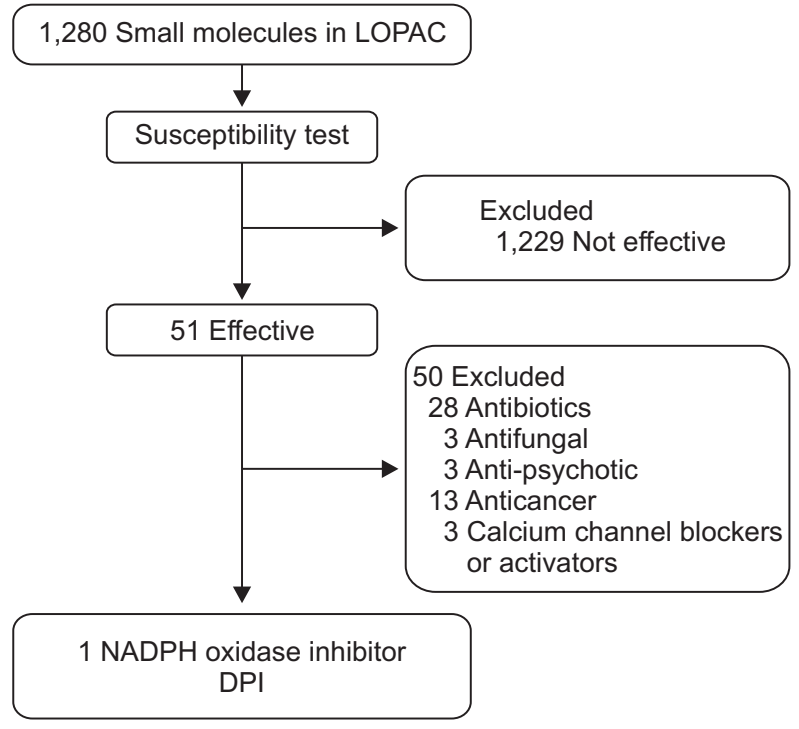

Fig. 1. Flow chart showing the selection of anti-Helicobacter pylori agents.

LOPAC, library of pharmacologically active compounds; NADPH, nicotinamide adenine dinucleotide phosphate; DPI, diphenyleneiodonium.

Table 1. Antimicrobial Susceptibilities of the Tested Helicobacter pylori Strains

\begin{tabular}{|c|c|c|c|c|c|c|}
\hline \multirow{2}{*}{ Strain } & \multirow{2}{*}{ Resistance } & \multicolumn{5}{|c|}{ MIC, $\mu \mathrm{g} / \mathrm{mL}$} \\
\hline & & CLA & AMO & MET & LEV & DPI \\
\hline HP43504 & MET & 0.03 & $<0.03$ & 128 & 0.25 & $<0.03$ \\
\hline CS_S1 & - & 0.03 & 0.125 & 4 & 0.25 & $<0.03$ \\
\hline CS_S2 & - & 0.03 & 0.125 & 4 & 0.25 & $<0.03$ \\
\hline CS_S3 & - & 0.03 & $<0.03$ & 2 & 0.25 & $<0.03$ \\
\hline CS_C1 & CLA & 64 & 0.25 & 4 & 0.5 & $<0.03$ \\
\hline CS_C2 & CLA & 32 & 0.125 & 4 & 0.5 & $<0.03$ \\
\hline CS_C3 & CLA & 64 & $<0.03$ & 2 & 0.25 & $<0.03$ \\
\hline CS_M1 & MET & 0.06 & $<0.03$ & 128 & 0.5 & $<0.03$ \\
\hline CS_M2 & MET & 0.06 & $<0.03$ & 128 & 0.5 & $<0.03$ \\
\hline CS_M3 & MET & 0.06 & 0.125 & 128 & 0.5 & $<0.03$ \\
\hline CS_L1 & LEV & 0.03 & 0.25 & 2 & 16 & $<0.03$ \\
\hline CS_L2 & LEV & 0.06 & 0.125 & 2 & 32 & $<0.03$ \\
\hline CS_L3 & LEV & 0.06 & $<0.03$ & 4 & 8 & $<0.03$ \\
\hline CS_A1 & AMO & 0.06 & 1 & 4 & 0.25 & $<0.03$ \\
\hline CS_A2 & AMO & 0.06 & 2 & 4 & 0.25 & $<0.03$ \\
\hline CS_A3 & AMO & 0.03 & 4 & 4 & 0.25 & $<0.03$ \\
\hline CS_CM1 & CLA/MET & 32 & $<0.03$ & 128 & 0.5 & $<0.03$ \\
\hline CS_CM2 & CLA/MET & 64 & $<0.03$ & 16 & 0.25 & $<0.03$ \\
\hline CS_CM3 & CLA/MET & 32 & 0.03 & 64 & 0.25 & $<0.03$ \\
\hline CS_CA1 & CLA/AMO & 64 & 1 & 2 & 0.25 & $<0.03$ \\
\hline CS_CA2 & CLA/AMO & 64 & 1 & 4 & 0.25 & $<0.03$ \\
\hline CS_CA3 & CLA/AMO & 32 & 2 & 1 & 0.25 & $<0.03$ \\
\hline CS_CML & CLA/MET/LEV & 128 & 0.06 & 128 & 64 & $<0.03$ \\
\hline CS_CMA & CLA/MET/AMO & 64 & 1 & 64 & 0.5 & $<0.03$ \\
\hline
\end{tabular}

MIC, minimal inhibitory concentration; CLA, clarithromycin; AMO, amoxicillin; MET, metronidazole; LEV, levofloxacin; DPI, diphenyleneiodonium; HP, Helicobacter pylori; CS, clinical strain. 
Table 2. Antimicrobial Susceptibility of Gram-Negative and Gram-Positive Pathogenic Bacteria to DPI and Vancomycin

\begin{tabular}{|c|c|c|c|}
\hline & \multirow{2}{*}{ Strain } & \multicolumn{2}{|c|}{ MIC, $\mu \mathrm{g} / \mathrm{mL}$} \\
\hline & & DPI & Vancomycin \\
\hline \multirow[t]{4}{*}{ Gram-positive } & Staphylococcus aureus 29213 (MSSA) & 1 & 1 \\
\hline & Staphylococcus aureus 33591 (MRSA) & 1 & 1 \\
\hline & Enterococcus faecalis 29212 & 1 & 4 \\
\hline & Staphylococcus epidermidis 12228 & 1 & 2 \\
\hline \multirow[t]{6}{*}{ Gram-negative } & Escherichia coli 25922 & 0.5 & $>32$ \\
\hline & Pseudomonas aeruginosa 27853 & 2 & $>32$ \\
\hline & Acinetobacter baumannii 19606 & 1 & $>32$ \\
\hline & Salmonella enteritidis 13076 & 1 & $>32$ \\
\hline & Salmonella typhimurium 13311 & 1 & $>32$ \\
\hline & Enterobacter cloacae 13049 & 1 & $>32$ \\
\hline
\end{tabular}

DPI, diphenyleneiodonium; MIC, minimal inhibitory concentration; MSSA, methicillin-sensitive Staphylococcus aureus; MRSA, methicillin-resistant Staphylococcus aureus.

\section{Antibacterial activity of DPI against common pathogenic bacteria}

The activity of DPI against common gram-negative and -positive pathogenic bacteria was also assessed. The MIC results are indicated in Table 2 and ranged between 0.5 and $2 \mu \mathrm{g} /$ $\mathrm{mL}$ against gram-negative bacteria. The MIC of DPI against all of the tested gram-positive bacteria was $1 \mu \mathrm{g} / \mathrm{mL}$. These MIC values are comparable to those of vancomycin, which is a commonly used antibiotic against gram-positive bacteria including methicillin-resistant Staphylococcus aureus.

\section{DISCUSSION}

We demonstrate that the NADPH oxidase inhibitor DPI has inhibitory activities against reference and resistant $H$. pylori strains and could be a candidate for further studies and future drug development. Importantly, the MIC value of DPI is lower than previously reported chemical compounds. ${ }^{22}$ The direct mechanism by which DPI inhibits $H$. pylori has not been clearly evaluated, but our indirect in vitro evidence suggests that DPI noncompetitively inhibits NADPH oxidase via covalent binding to flavin adenine dinucleotide. Although DPI is a nonspecific inhibitor of flavoenzymes, a decrease in ROS production has been reported in DPI-treated cells. ${ }^{23}$ Based on this decrease in ROS production, it has been speculated that DPI may reverse atherosclerosis and this compound is now under investigation to determine its neuroprotective effects in focal cerebral ischemia, a neurodegenerative disease. ${ }^{24}$ Micromolar concentrations of DPI are highly toxic, but sub-picomolar concentrations of DPI inhibit NADPH oxidase activation with high specificity. ${ }^{25}$ In another study, DPI was reported to inhibit $H$. pylori-induced increases in ROS, NADPH oxidase activity, MCP-1 expression, and the activation of MAPKs (mitogen-activated protein ki- nases), including the extracellular signal-regulated kinases, p38, and Jun N-terminal kinases, in AGS cells. ${ }^{26}$

DPI is likely to be a novel agent in the future for two reasons. First, a NADPH oxidase inhibitor is a new substance that has never been used to treat $H$. pylori infection. Previous studies have shown that some chemicals inhibit activation of NADPH oxidase, suggesting that they may alleviate cell inflammation in H. pylori-infected gastric epithelial cells. ${ }^{27,28}$ DPI may also suppress unnatural apoptosis in gastric epithelial cells infected with $H$. pylori. ${ }^{29}$ However, whether DPI can directly eradicate $H$. pylori has not been reported. In our in vitro study, we demonstrated that DPI effectively eradicated $H$. pylori. This is the first study to investigate the efficacy of DPI for eradicating $H$. pylori. This novel agent may be free from current antibiotic resistance, so it may help to overcome antibiotic resistance. Second, NADPH oxidase exists in gastric mucosal tissue, and DPI is a nonselective inhibitor of NADPH oxidase. ${ }^{30,31} H$. pylori infections increase ROS in infected cells using NADPH oxidase. This increase in ROS causes oxidative DNA damage in infected cells and may provoke $H$. pylori-infected carcinogenesis. ${ }^{32}$ Therefore, a substance with a NADPH oxidase inhibitor mechanism, such as DPI, may inhibit carcinogenesis and eradicate $H$. pylori. Finally, in this study, DPI was effective against all $H$. pylori strains regardless of antibiotic resistance. Considering these findings, we expect that DPI may help to overcome multidrug-resistant $H$. pylori in the future.

As mentioned earlier, the rate of resistance to commonly used antibiotics such as clarithromycin, metronidazole, and fluoroquinolone is the main cause of the decrease in the eradication rate of certain bacteria. In addition, the number of multidrugresistant bacterial strains has increased. This highlights a critical need to develop selective antibacterial agents with novel target sites and establish an effective drug-resistance management strategy. In contrast, the development of new antimicrobial 
agents is somewhat out of proportion. Rifabutin, furazolidone, sitafloxacin, and nitazoxanide have been introduced but are not always available in some countries. In addition, rifabutin demonstrates serious side effects, such as myelosuppression, and should be reserved to treat mycobacterial infections. Furazolidone is a nitrofuran antibiotic that has demonstrated efficacy in some trials. However, it has been recognized by the U.S. Food and Drug Administration as a carcinogenic agent and thus is no longer used, except in a few developing countries. ${ }^{33}$ Sitafloxacin seems to be effective, but clinical data are still limited in Japan. ${ }^{34,35}$ Nitazoxanide is an antiprotozoal agent that has demonstrated efficacy in a restricted study, but it is a somewhat expensive agent. ${ }^{36,37}$ Further studies of these antibiotics are needed.

In addition to these antimicrobial approaches, therapeutic alternatives beyond antibiotics have been investigated in recent years, including natural phytotherapy and probiotics. ${ }^{22,38}$ Representative agents include Lactobacillus reuteri, Korean red ginseng, and sulforaphane. Micro- and nano-technologies have also been used to develop gastric drug delivery systems. ${ }^{39,40}$

Acid stability is an important factor for antibiotics used to treat $H$. pylori. Some studies have reported that major antibiotics (clarithromycin, amoxicillin, metronidazole) for $H$. pylori treatment degrade in human gastric acid at different $\mathrm{pH}$ values. Metronidazole is stable in an acidic environment (half-life $>800$ hours); however, clarithromycin is unstable in an acidic environment (half-life $<1$ hour). ${ }^{41,42}$ The quick destruction of antibiotics in gastric acid makes them less effective at eradicating $H$. pylori. Therefore, it is important that this problem is solved. No study has reported on the influence of gastric juice on antibacterial efficacy and acid stability of DPI. Additionally, DPI has not been reported as an antibiotic in humans. However, DPI has excellent antibiotic effects in vitro, and it has an antimicrobial effect on $H$. pylori as well as other gram-negative and -positive pathogenic bacteria. DPI is also expected to maintain acid stability using these methods, but more studies are needed.

We demonstrated that DPI has the ability to strongly eradicate $H$. pylori. Although DPI is relatively highly toxic, some studies have reported that ultra-low dose DPI is not overtly toxic in mice and has potent neuroprotective efficacy. ${ }^{24,43}$ Therefore, we could produce a substance that strongly inhibits $H$. pylori infection without toxic side effects by identifying the proper dose of DPI to treat $H$. pylori. Additionally, DPI has excellent antibacterial effects even in multidrug-resistant $H$. pylori. These advantages could help DPI serve as a novel agent in the future. Although we identified a novel $H$. pylori chemical inhibitor, additional steps are needed to develop such a substance into a viable drug because of several limitations. First, DPI is a nonspecific NADPH inhibitor, so drug development is somewhat limited. Second, the interactions between DPI and other medications (PPIs and other antibiotics) for treating $H$. pylori have not been elucidated. Finally, the effects of an acidic environment on the activities of anti- $H$. pylori agent have not yet been consid- ered. The high environmental $\mathrm{pH}$ of the stomach may affect the susceptibility of these bacteria to antibiotics. Further research is needed to examine the effects of $\mathrm{pH}$ on anti- $H$. pylori activity in different agents. Animal experiments will also be needed to determine whether substances such as DPI are effective under in vivo conditions.

In conclusion, DPI shows a potent MIC value against $H$. pylori, suggesting that it might be a promising new agent for that could be used to significantly improve anti-H. pylori treatment success and eradicate this pathogen.

\section{CONFLICTS OF INTEREST}

No potential conflict of interest relevant to this article was reported.

\section{ACKNOWLEDGEMENTS}

This work was supported by a grant from the Asan Institute for Life Sciences (number: 2013-348, Asan Medical Center, Seoul, Korea) and Il-Yang Pharmaceutical (GCU 2014-5115).

\section{REFERENCES}

1. Hentschel E, Brandstätter G, Dragosics B, et al. Effect of ranitidine and amoxicillin plus metronidazole on the eradication of Helicobacter pylori and the recurrence of duodenal ulcer. N Engl J Med 1993;328:308-312.

2. Suerbaum S, Josenhans C. Helicobacter pylori evolution and phenotypic diversification in a changing host. Nat Rev Microbiol 2007;5:441-452.

3. Chung JW, Lee GH, Han JH, et al. The trends of one-week firstline and second-line eradication therapy for Helicobacter pylori infection in Korea. Hepatogastroenterology 2011;58:246-250.

4. Graham DY, Fischbach L. Helicobacter pylori treatment in the era of increasing antibiotic resistance. Gut 2010;59:1143-1153.

5. Gisbert JP, Calvet X. Review article: non-bismuth quadruple (concomitant) therapy for eradication of Helicobater pylori. Aliment Pharmacol Ther 2011;34:604-617.

6. Chung JW, Jung YK, Kim YJ, et al. Ten-day sequential versus triple therapy for Helicobacterpylori eradication: a prospective, openlabel, randomized trial. J Gastroenterol Hepatol 2012;27:16751680.

7. Chung JW, Ha M, Yun SC, et al. Meta-analysis: sequential therapy is superior to conventional therapy for Helicobacter pylori infection in Korea. Korean J Gastroenterol 2013;62:267-271.

8. Malfertheiner P, Megraud F, O'Morain CA, et al. Management of Helicobacter pylori infection: the Maastricht IV/ Florence consensus report. Gut 2012;61:646-664.

9. Ierardi E, Giorgio F, Losurdo G, Di Leo A, Principi M. How antibiotic resistances could change Helicobacter pylori treatment: a matter of geography? World J Gastroenterol 2013;19:8168-8180. 
10. Kim SY, Choi DJ, Chung JW. Antibiotic treatment for Helicobacter pylori: is the end coming? World J Gastrointest Pharmacol Ther 2015;6:183-198.

11. Molina-Infante J, Shiotani A. Practical aspects in choosing a Helicobacter pylori therapy. Gastroenterol Clin North Am 2015;44:519535.

12. Jeong MH, Chung JW, Lee SJ, et al. Comparison of rifabutin- and levofloxacin-based third-line rescue therapies for Helicobacter pylori. Korean J Gastroenterol 2012;59:401-406.

13. Heep M, Rieger U, Beck D, Lehn N. Mutations in the beginning of the rpoB gene can induce resistance to rifamycins in both Helicobacter pylori and Mycobacterium tuberculosis. Antimicrob Agents Chemother 2000;44:1075-1077.

14. Navarro-Rodriguez T, Silva FM, Barbuti RC, et al. Association of a probiotic to a Helicobacter pylori eradication regimen does not increase efficacy or decreases the adverse effects of the treatment: a prospective, randomized, double-blind, placebo-controlled study. BMC Gastroenterol 2013;13:56.

15. Mirzaee V, Rezahosseini 0. Randomized control trial: comparison of triple therapy plus probiotic yogurt vs. standard triple therapy on Helicobacter pylori eradication. Iran Red Crescent Med J 2012;14:657666.

16. Lee JW, Kim N, Kim JM, et al. Prevalence of primary and secondary antimicrobial resistance of Helicobacter pylori in Korea from 2003 through 2012. Helicobacter 2013;18:206-214.

17. Watamoto T, Egusa H, Sawase T, Yatani H. Screening of pharmacologically active small molecule compounds identifies antifungal agents against Candida biofilms. Front Microbiol 2015;6:1453.

18. Altaf M, Miller CH, Bellows DS, O'Toole R. Evaluation of the Mycobacterium smegmatis and BCG models for the discovery of Mycobacterium tuberculosis inhibitors. Tuberculosis (Edinb) 2010;90:333-337.

19. Lucumi E, Darling C, Jo H, et al. Discovery of potent small-molecule inhibitors of multidrug-resistant Plasmodium falciparum using a novel miniaturized high-throughput luciferase-based assay. Antimicrob Agents Chemother 2010;54:3597-3604.

20. Che P, Wang L, Li Q. The development, optimization and validation of an assay for high throughput antiviral drug screening against Dengue virus. Int J Clin Exp Med 2009;2:363-373.

21. Jean B. Performance standards for antimicrobial susceptibility testing: 25th informational supplement. Wayne: Clinical and Laboratory Standards Institute, 2015.

22. Makobongo MO, Gilbreath JJ, Merrell DS. Nontraditional therapies to treat Helicobacter pylori infection. J Microbiol 2014;52:259272.

23. Bhunia AK, Han H, Snowden A, Chatterjee S. Redox-regulated signaling by lactosylceramide in the proliferation of human aortic smooth muscle cells. J Biol Chem 1997;272:15642-15649.

24. Wang Q, Chu CH, Oyarzabal E, et al. Subpicomolar diphenyleneiodonium inhibits microglial NADPH oxidase with high specificity and shows great potential as a therapeutic agent for neurodegenerative diseases. Glia 2014;62:2034-2043.
25. Aldieri E, Riganti C, Polimeni M, et al. Classical inhibitors of NOX NAD(P)H oxidases are not specific. Curr Drug Metab 2008;9:686696

26. Cho SO, Lim JW, Kim KH, Kim H. Diphenyleneiodonium inhibits the activation of mitogen-activated protein kinases and the expression of monocyte chemoattractant protein-1 in Helicobacter pylori-infected gastric epithelial AGS cells. Inflamm Res 2011;60:501-507.

27. Cha B, Lim JW, Kim KH, Kim H. 15-Deoxy-D12,14-prostaglandin J2 suppresses RANTES expression by inhibiting NADPH oxidase activation in Helicobacter pylori-infected gastric epithelial cells. J Physiol Pharmacol 2011;62:167-174.

28. Cho SO, Lim JW, Kim H. Red ginseng extract inhibits the expression of MCP-1 and iNOS in Helicobacter pylori-infected gastric epithelial cells by suppressing the activation of NADPH oxidase and Jak2/Stat3. J Ethnopharmacol 2013;150:761-764.

29. Cho SO, Lim JW, Kim H. Diphenyleneiodonium inhibits apoptotic cell death of gastric epithelial cells infected with Helicobacter pylori in a Korean isolate. Yonsei Med J 2015;56:1150-1154.

30. Tominaga K, Kawahara T, Sano T, et al. Evidence for cancerassociated expression of NADPH oxidase 1 (Nox1)-based oxidase system in the human stomach. Free Radic Biol Med 2007;43:16271638.

31. Jones RD, Hancock JT, Morice AH. NADPH oxidase: a universal oxygen sensor? Free Radic Biol Med 2000;29:416-424.

32. Jang SH, Lim JW, Morio T, Kim H. Lycopene inhibits Helicobacter pylori-induced ATM/ATR-dependent DNA damage response in gastric epithelial AGS cells. Free Radic Biol Med 2012;52:607-615.

33. World Gastroenterology Organisation. World Gastroenterology Organisation global guideline: Helicobacter pylori in developing countries. J Clin Gastroenterol 2011;45:383-388.

34. Furuta T, Sugimoto M, Kodaira C, et al. Sitafloxacin-based thirdline rescue regimens for Helicobacter pylori infection in Japan. J Gastroenterol Hepatol 2014;29:487-493.

35. Murakami K, Furuta T, Ando T, et al. Multi-center randomized controlled study to establish the standard third-line regimen for Helicobacter pylori eradication in Japan. J Gastroenterol 2013;48:1128-1135.

36. Basu PP, Rayapudi K, Pacana T, Shah NJ, Krishnaswamy N, Flynn M. A randomized study comparing levofloxacin, omeprazole, nitazoxanide, and doxycycline versus triple therapy for the eradication of Helicobacter pylori. Am J Gastroenterol 2011;106:19701975.

37. Watson JB, Moss SF. Will H. pylori stagger under the weight of this LOAD? A novel but expensive eradication regimen. Am J Gastroenterol 2011;106:1976-1977.

38. Vale FF, Oleastro M. Overview of the phytomedicine approaches against Helicobacter pylori. World J Gastroenterol 2014;20:55945609.

39. Lopes D, Nunes C, Martins MC, Sarmento B, Reis S. Eradication of Helicobacter pylori: past, present and future. J Control Release 2014;189:169-186 
654 Gut and Liver, Vol. 11, No. 5, September 2017

40. Gao W, Thamphiwatana S, Angsantikul P, Zhang L. Nanoparticle approaches against bacterial infections. Wiley Interdiscip Rev Nanomed Nanobiotechnol 2014;6:532-547.

41. Erah PO, Goddard AF, Barrett DA, Shaw PN, Spiller RC. The stability of amoxycillin, clarithromycin and metronidazole in gastric juice: relevance to the treatment of Helicobacter pylori infection. J Antimicrob Chemother 1997;39:5-12.
42. Labenz J. Current role of acid suppressants in Helicobacter pylori eradication therapy. Best Pract Res Clin Gastroenterol 2001;15:413-431.

43. Wang Q, Qian L, Chen SH, et al. Post-treatment with an ultra-low dose of NADPH oxidase inhibitor diphenyleneiodonium attenuates disease progression in multiple Parkinson's disease models. Brain 2015;138(Pt 5):1247-1262. 